\title{
Aplicação da metodologia Box-Jenkins para previsão do ICMS do estado do Paraná de agosto de 2011 a julho de 2012
}

Ademir Clemente*

Leonel Toshio Clemente*

\begin{abstract}
RESUMO - Considerando a importância da arrecadação de ICMS para o orçamento do governo do Paraná e a necessidade de previsões para o planejamento das despesas, busca-se, neste artigo, prever a arrecadação deste imposto para o período de agosto de 2011 a julho de 2012. Para tanto, aplicou-se a metodologia Box-Jenkins para modelar a série mensal de ICMS de janeiro de 2000 a julho de 2011, a qual foi diferenciada para tornar-se estacionária. A partir da análise de resíduo e dos testes de significância dos parâmetros, elegeu-se a identificação SARIMA $(2,1,0) \times(0,1,1) 12$ para previsão. De acordo com este modelo, a arrecadação de ICMS crescerá 13,2\% entre julho de 2011 e janeiro de 2012, diminuirá 5\% entre janeiro e fevereiro de 2012, e aumentará $6,7 \%$ entre fevereiro e julho de 2012.
\end{abstract}

Palavras-chave: Metodologia Box-Jenkins. Previsão. ICMS. Paraná.

\section{INTRODUÇÃO}

O Imposto Sobre Circulação de Mercadorias e Serviços (ICMS), criado na reforma constitucional de 1988, é o tributo de maior arrecadação no Brasil (REZENDE, 2009), incide sobre o valor adicionado, tem caráter plurifásico, não cumulativo, de competência decisória estadual e periodicidade mensal.

Por ser o tributo de maior arrecadação no Brasil, autores como Guaragna e Mello (2002), Corvalão (2002), Camargos (2008), Liebel (2004, 2005), Marques e Uchôa (2006), Santos e Costa (2008), buscaram prever econometricamente sua arrecadação. Guaragna e Mello (2002), assim como Camargos (2008), em seus estudos de previsão, justificam a utilização de modelos univariados tendo em vista que a base de dados imponível do ICMS é não observável. Marques e Uchôa (2006, p. 199) ressaltam que a utilização de modelos causais, como os de Corvalão (2002), se utilizarem proxies inadequadas para a base imponível, como o PIB, geram estimadores viesados e ineficientes. Utilizando modelos de alisamento exponencial, Santos e Costa (2008) fazem a previsão do ICMS do Maranhão. Porém, como reconhecem estes autores, a utilização de modelos da classe ARIMA pode gerar melhores previsões.

\footnotetext{
" Doutor em Engenharia de Transportes pela Universidade Federal do Rio de Janeiro. É professor adjunto da Universidade Federal do Paraná. Endereço eletrônico: ademir@ufpr.br.

** Graduado em Ciências Econômicas pela Universidade Federal do Paraná. Mestrando em Desenvolvimento Econômico pelo Programa de Pós-Graduação em Economia da Universidade Federal do Rio Grande do Sul. É bolsista da CAPES/CNPq. Endereço eletrônico: leonel_t_clemente@hotmail.com.
} 
Autores como Passos et al. (2011), Castanho (2011) e Cruz (2007) utilizaram modelos da classe ARIMA para a previsão do ICMS dos estados do Pará, Espírito Santo e Piauí, respectivamente. Liebel $(2004,2005)$ realizou previsões a partir de modelos univariados, dentre eles os modelos da classe ARIMA, especificamente para o ICMS do estado do Paraná. Os estudos de Liebel são os únicos publicados que modelam a arrecadação de ICMS do estado do Paraná utilizando a metodologia Box-Jenkins, porém seu horizonte de previsão é 2004.

A atualização das previsões de receita do ICMS é absolutamente prioritária para a Coordenação da Receita do Estado do Paraná ${ }^{1}$, pois, para este estado, o ICMS correspondeu, em média, a $91 \%$ das receitas tributárias e 89\% das receitas correntes durante o período de 1995 a 2009 (STN, 2010).

Considerando a importância do ICMS para o orçamento do governo paranaense e a necessidade de previsões de arrecadação para o planejamento das despesas, busca-se realizar uma previsão da arrecadação deste imposto para um horizonte de doze meses a partir do último dado, referente a julho de 2011. Para tanto, aplicou-se a metodologia Box-Jenkins para modelar a série mensal de ICMS de janeiro de 2000 a julho de 2011.

Este artigo está organizado em 6 seções, além desta introdução. Realiza-se na Seção 2 uma revisão bibliográfica dos estudos de projeção de arrecadação do ICMS com utilização da metodologia Box-Jenkins. A Seção 3 apresenta uma breve discussão acerca dos fatores que causaram as variações mais importantes no período de 1994 a 2011. A Seção 4 é constituída de notas metodológicas referentes à estimação de modelos baseados na metodologia Box-Jenkins. Descreve-se a série utilizada e apresentam-se os testes de estacionariedade. $\mathrm{Na}$ Seção 5, relata-se o processo de identificação do modelo SARIMA (p,d,q)x(P,D,Q) para a série de ICMS diferenciada. Na Seção 6, faz-se a previsão da série de ICMS a partir do modelo SARIMA $(2,1,0) x(0,1,1) 12$ para o período de agosto de 2011 a julho de 2012. Na seção seguinte, apresentam-se as principais conclusões.

\section{ESTUDOS DE PROJEÇÃO DE ARRECADAÇÃO DE ICMS COM EMPREGO DA METODOLOGIA BOX-JENKINS}

Nesta seção revisam-se brevemente os principais estudos que aplicaram a metodologia Box-Jenkins para a projeção de arrecadação de ICMS.

Passos et al. (2011) realizaram estudo para o estado do Pará abrangendo o período de 1992 a 2002 e utilizando a arrecadação de ICMS deflacionada pelo IGP-DI. Aplicando o teste

1 A relevância das previsões da arrecadação do ICMS, reconhecida pela Coordenação da Receita do Estado do Paraná, orientou a Lei de Diretrizes Orçamentárias de 2006 (LIEBEL, 2005, p. 8). 
de Ljung-Box e o critério de informação de Akaike, os pesquisadores elegeram o SARIMA $(0,1,1) \times(0,1,1) 12$ como o modelo mais adequado para previsão.

Cruz (2007) também trabalhou com dados de arrecadação de ICMS deflacionados pelo IGP-DI. A partir do teste Dickey-Fuller Aumentado (ADF), constatou estacionariedade em nível. Sem ter realizado testes estatísticos para sazonalidade e raiz unitária sazonal, o referido autor simulou modelos ARMA (p,q) para a arrecadação de ICMS do Piauí no período de 1996 a 2006 alternativamente com e sem dummy para quebra estrutural. Então, selecionou para previsão sem quebra estrutural o ARMA $(1,0)$ e, com quebra, o ARMA $(2,3)$. A escolha destes modelos teve como base o critério do erro absoluto percentual médio e, por consequência, o ARMA (2,3) foi considerado o melhor modelo para previsão.

Para o estado do Espírito Santo, Castanho (2011) aplicou logaritmo à série de ICMS nominal de 2000 a 2009 e modelou-a como um processo ARIMA $(1,0,2)$.

Liebel (2004) empregou a metodologia Box-Jenkins para previsão da arrecadação de ICMS do estado do Paraná. Os dados de arrecadação de ICMS são nominais e se referem ao período de janeiro de 1997 a dezembro de 2002. Pelo critério do erro percentual médio, o autor identificou e utilizou para previsão o modelo SARIMA $(1,1,0) \mathrm{x}(2,0,1) 12$.

\section{VARIAÇÕES OBSERVADAS NA SÉRIE DE ICMS DO PARANÁ E SUAS CAUSAS}

O aumento da arrecadação do ICMS no Paraná entre 1994 e 2011 decorreu, em grande parte, do crescimento das atividades industriais, processo que teve início em 1985. Houve crescimento de 101,8\% na capacidade instalada da indústria paranaense entre 1990 e 1995, e aumento de 14\% entre 1995 e 2000 (IPARDES, 2002). O deslocamento da série de arrecadação do ICMS para o novo patamar somente ocorreu após alguns anos de aumento da capacidade instalada, pois o governo paranaense, como incentivo fiscal, concedeu isenção temporária para os principais novos empreendimentos.

Observando o movimento das séries que compõem o ICMS, há indícios de que o crescimento industrial paranaense continuou depois de 2000, como se pode visualizar no Gráfico 1.

A arrecadação de ICMS apresentou variações que acompanhavam as variações do PIB entre 1994 e 1997. Em 1997, houve uma queda em termos reais da arrecadação, resultado da desoneração das operações de exportação de produtos básicos e semimanufaturados, em decorrência da Lei Complementar 87/96 (Lei Kandir), que entrou em vigor em setembro de 1996. Como muitas Unidades da Federação tiveram significativas reduções na arrecadação do ICMS, a União criou um fundo provisório para repasse de valores aos mais prejudicados. 
Porém, estes repasses foram reduzidos ao longo dos primeiros anos de vigência da referida lei (LIEBEL, 2004).
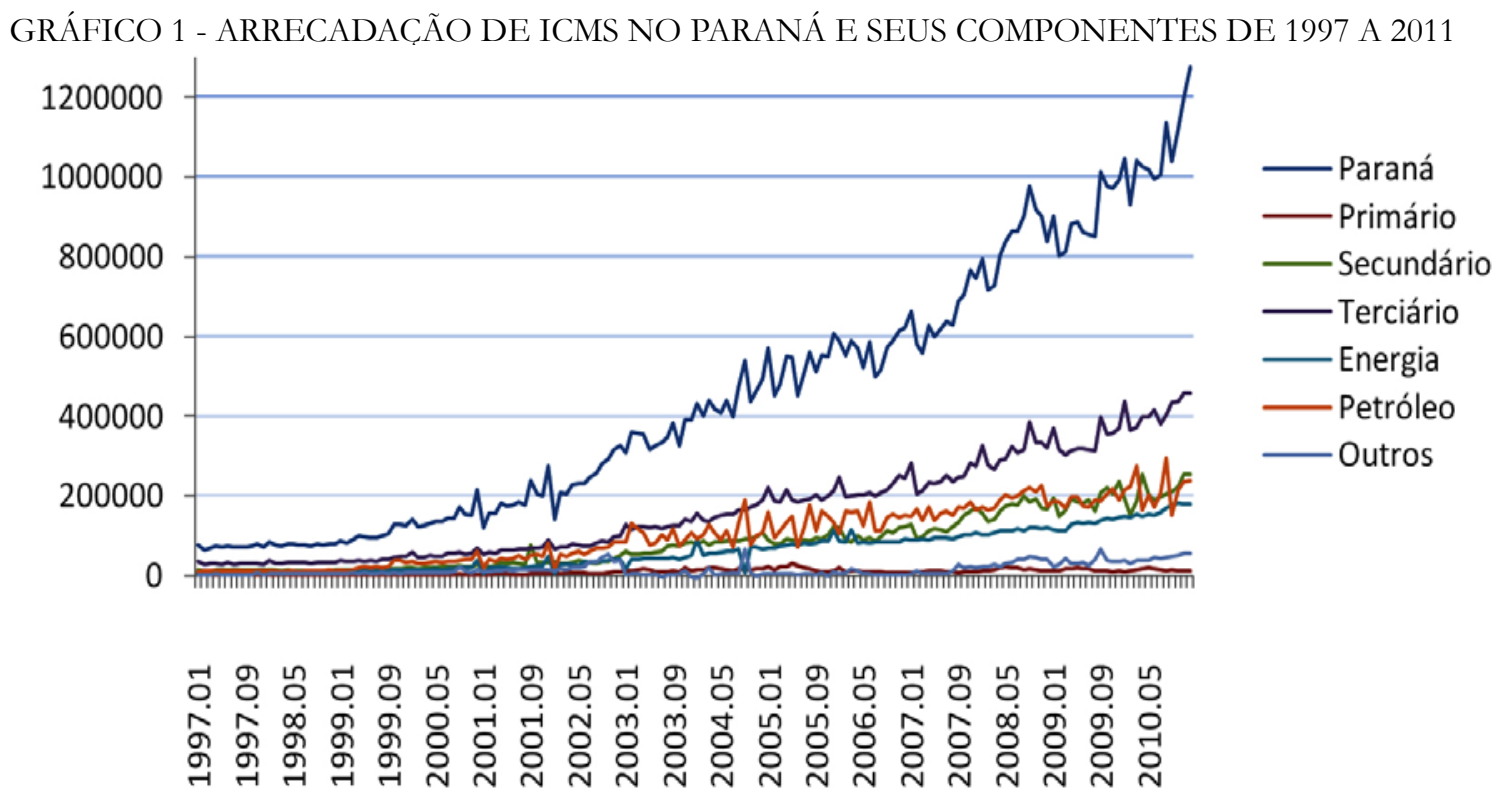

FONTE: IPEADATA (2011b).

NOTA: Dados em mil reais deflacionados pelo IGP-DI (julho de $2011=100 \%$ ).

O aumento significativo da arrecadação em 1999 pode estar relacionado às implantações de novos sistemas integrados e novas formas de gerenciamento de informações online pela Receita Estadual. Com este instrumental moderno, ocorreu ganho de eficácia das atividades de auditoria fiscal, com decorrente redução de evasão fiscal (LIEBEL, 2004, p. 73).

Em 2002, observa-se aumento na arrecadação do ICMS como decorrência da promulgação da Lei 13.410, em 26 de dezembro de 2001. A nova lei aumentou as alíquotas de ICMS: nas operações internas, aumento de $17 \%$ para 18\%. Sobre gasolina e álcool combustível, aumento de $25 \%$ para $26 \%$. Sobre energia elétrica, bebidas alcoólicas, fumo e prestação de serviços de comunicação a alíquota aumentou de 25\% para 27\% (LIEBEL, 2004, p. 74).

Observando a série de arrecadação do ICMS no Gráfico 1, notam-se indícios de que há sazonalidade na série. Essa hipótese tem sustentação lógica, pois as atividades econômicas de praticamente todos os setores apresentam variações sazonais. Também se pode notar que a variância da série aumentou ao longo do tempo. Isso, no entanto, pode estar relacionado com o simples aumento, em termos absolutos, da arrecadação ao longo do tempo.

\section{NOTAS METODOLÓGICAS}

Esta seção resume as principais características da metodologia de previsão conhecida como abordagem de Box-Jenkins (1970), apresenta os testes de estacionariedade e descreve 
sucintamente os dados utilizados.

\subsection{A METODOLOGIA BOX-JENKINS}

A metodologia Box-Jenkins (1970) toma por base o Teorema de Wald e consiste em ajustar modelos autorregressivos integrados de médias móveis a um conjunto de dados. A modelagem é baseada em um ciclo iterativo, em que a escolha da estrutura do modelo é endógena, ou seja, a partir dos dados (MORETTIN; TOLOI, 2006, p. 105).

Os estágios deste ciclo iterativo consistem em: especificação, identificação, estimação e verificação. No estágio de especificação, uma classe genérica de modelos é considerada para a análise. Na segunda fase, identifica-se o modelo. Para isso, analisa-se a função de autocorrelação (FAC) e a função de autocorrelação parcial (FACP). Na fase de estimação do modelo, os parâmetros identificados na fase anterior são estimados. A última fase consiste em testar o modelo, ou seja, fazer um diagnóstico do modelo ajustado. Para tanto, analisa-se o resíduo da regressão estimada e verifica-se a capacidade de previsão do modelo. Se o modelo for considerado adequado, poderá ser utilizado para previsão; caso contrário, deve-se recomeçar todo o processo. $\mathrm{Na}$ prática, admitem-se, desde início, várias identificações alternativas, as quais são testadas e comparadas. Tendo em vista a presença de sazonalidade, foram especificados modelos da classe SARIMA.

\subsection{TESTES DE ESTACIONARIEDADE}

$\mathrm{Na}$ metodologia Box-Jenkins, para poder estimar os parâmetros do modelo autorregressivo de médias móveis, deve-se verificar se a série é estacionária. Caso não seja, deve-se torná-la estacionária.

Para testar esta propriedade, utilizaram-se duas versões do teste originalmente desenvolvido por Dickey e Fuller. Para testar a parte não sazonal, realizou-se o teste Dickey-Fuller Aumentado (ADF), e para a parte sazonal, utilizou-se o teste Dickey-Hasza-Fuller (1984). O teste Dickey-Hasza-Fuller (DHF) faz parte da família dos testes Dickey-Fuller, os quais são baseados na análise do estimador do parâmetro autoregressivo de várias ordens (MONTAÑÉS; SANSÓ, 2001, p. 74).

O teste ADF consiste em estimar os parâmetros autoregressivos de várias ordens e testar a presença de raiz unitária a partir da comparação da estatística $t$ com a estatística DickeyFuller. O teste Dickey-Hasza-Fuller utiliza apenas as variáveis com a defasagem sazonal e tem as mesmas hipóteses das outras variações do teste, sendo a hipótese nula a presença de raiz unitária. Porém, o teste DHF segue a distribuição apresentada em Dickey, Hazsa, Fuller (1984, 
p. 362).

\subsection{DADOS UTILIZADOS}

Os dados de arrecadação de ICMS do estado do Paraná foram coletados em IPEADATA (2011b), e deflacionadas pelo IGP-DI (IPEADATA, 2011a). Conforme o Gráfico 1, há indícios de que a arrecadação apresenta mudança de tendência a partir do ano 2000, e de que sua variância passa a ser maior. Isso pôde ser confirmado por meio do teste $\mathrm{F}$ (com 5\% de significância) comparando as variâncias da série em dois subperíodos, 1994 a 2000, e 2001 a 2011. O aumento da variância pode ser resultado do aumento do valor absoluto da arrecadação de ICMS no tempo; pois, regredindo a variância de cada doze meses contra o tempo e tempo ao quadrado, desde junho de 1997 até janeiro de 2010, obtiveram-se coeficientes significativos a $5 \%$.

Dessa forma, considerando as mudanças na base de cálculo que entraram em vigor em setembro de 1996, e a mudança tecnológica pela qual passou o sistema de arrecadação tributário paranaense em 1999, optou-se por regredir o modelo utilizando dados entre janeiro de 2001 e julho de 2011.

\section{IDENTIFICAÇÃO DO MODELO SARIMA}

Por meio do software Eviews (2004), testou-se a estacionariedade da série do ICMS do Paraná. No teste ADF foram inseridas as defasagens de 1 a 6 , e obteve-se rejeição da hipótese de raiz unitária a partir da primeira diferença (a 5\% de significância). O teste ADF em nível calculou a estatística ADF igual a -1,34, que é um valor que não possibilita rejeitar a hipótese nula a 5\%. Por outro lado, como mostra a Tabela 1, a hipótese nula é rejeitada aplicando-se o teste $\mathrm{ADF}$ à série em primeira diferença, utilizando o máximo de 6 defasagens para os termos autorregressivos.

TABELA 1 - TESTE ADF PARA A SÉRIE DE ICMS PARANAENSE

\begin{tabular}{|c|c|r}
\hline \multicolumn{3}{|c}{ Hipótese nula: D (ICMS) tem raiz unitária } \\
\hline & Estatística t & \multicolumn{1}{c}{$p$-valor } \\
\hline Augmented Dickey-Fuller test statistic & -6.485885 & 0.0000 \\
\hline
\end{tabular}
FONTE: Saída do software Eviews 5.0 (2004).

Para a raiz unitária sazonal, aplicou-se o teste $\mathrm{DHF}^{2}$ e detectou-se que a série ICMS tem raiz sazonal, pois a estatística DHF calculada foi de $-2,97$, e não rejeita a hipótese nula quando comparada ao valor crítico de -5.86 (a 5\% de significância, amostra de 120 elementos e sazonalidade de 12 meses) calculado por Dickey, Hazsa, Fuller (1984, p. 362). Portanto, aplicou2 O teste foi especificado conforme Pierce (2011, p. 8-9) e Fomby (2011, p. 3). 
se o operador de defasagem:

$$
Z_{t}=(1-L)\left(1-L^{12}\right) Y_{t}
$$

onde $Z_{t}=$ série diferenciada (D1D12_ICMS), $Y_{t}=$ série de ICMS em nível, $L=$ Operador de defasagem.

Assim, obteve-se a série nomeada por D1D12_ICMS. Para esta série realizou-se o teste DHF, que gerou um valor $t$ calculado de $-8,45$, o qual, comparado ao valor tabelado de -5.86 (DICKEY, HAZSA, FULLER, 1984, p. 362), rejeitou a hipótese nula de raiz unitária com $95 \%$ de confiança.

TABELA 2 - TESTE DHF PARA A SÉRIE DE D1D12_ICMS

\begin{tabular}{lrrrrr}
\hline \multicolumn{5}{c}{ Variável dependente: (D1D12_ICMS)-(D1D12_ICMS(-12)) } \\
\hline \multicolumn{1}{c}{ Variável } & Coeficiente & Erro-padrão & Estatística $t$ & $p$-valor \\
\hline D1D12_ICMS(-12) & -1.462202 & 0.173035 & -8.450302 & 0.0000 \\
D(D1D12_ICMS(-12)) & -0.136808 & 0.111580 & -1.226099 & 0.2231 \\
D(D1D12_ICMS(-24)) & -0.210627 & 0.074932 & -2.810900 & 0.0060 \\
D(D1D12_ICMS(-36)) & -0.273246 & 0.075873 & -3.601363 & 0.0005 \\
D(D1D12_ICMS(-48)) & -0.204793 & 0.069958 & -2.927387 & 0.0042 \\
D(D1D12_ICMS(-56)) & 0.156927 & 0.065183 & 2.407495 & 0.0179 \\
\hline
\end{tabular}

FONTE: Saída do software Eviews 5.0 (2004).

A identificação das possíveis ordens dos termos AR e MA resultou da análise gráfica das funções de autocorrelação (FAC) e autocorrelação parcial (FACP) da série D1D12_ICMS. Assim, foram testados e comparados os modelos apresentados na Tabela 3.

TABELA 3 - COMPARAÇÃO DE MODELOS SARIMAS

\begin{tabular}{lr|r}
\hline \multicolumn{1}{c|}{ Modelos SARIMA } & \multicolumn{1}{c}{ MAE } & Significância \\
\hline (A) $(2,1,0) \times(0,1,1) 12$ com constante & 32304,1 & $*$ \\
(B) $(1,1,1) \times(1,1,1) 12$ com constante & 31690 & $* *$ \\
(C) $(1,1,2) \times(1,1,2) 12$ com constante & 31480,9 & $* *$ \\
(D) $(2,1,1) \times(2,1,1) 12$ com constante & 30314,7 & $* *$ \\
(E) $(2,1,2) \times(2,1,2) 12$ com constante & 30439,9 & $* *$ \\
(F) $(1,1,0) \times(0,1,1) 12$ com constante & 33484,2 & $*$ \\
(G) $(1,1,0) \times(0,1,2) 12$ com constante & 33554,1 & $* *$ \\
(H) $(2,1,0) \times(0,1,2) 12$ com constante & 32254,6 & $* *$ \\
\hline
\end{tabular}

FONTE: Elaboração a partir da saída do software Statgraphics (2007).

NOTA: $\left(^{*}\right)$ todos os coeficientes foram significativos a $5 \%$; $\left(^{*}\right)$ pelo menos um dos coeficientes não foi significativo a $5 \%$.

Apenas os modelos A e F são considerados relevantes. Analisando o periodograma acumulado do modelo F, observou-se indício de padrão sistemático no resíduo. Este indício foi reforçado pela análise da função de autocorrelação de resíduo, que apresentou correlação significativa a $5 \%$ na segunda defasagem.

Por outro lado, o modelo A, SARIMA $(2,1,0) \times(0,1,1) 12$, com constante, não apresen- 
tou indícios de padrão sistemático no resíduo. Pôde-se confirmar que o resíduo se caracteriza como ruído branco a partir da FAC, da FACP, da função de autocorrelação do resíduo ao quadrado e do teste Ljung-Box a 5\% de significância. Para aprofundar a análise, aplicou-se o teste LM de Breusch-Godfrey para autocorrelação serial, o qual não indicou nenhuma correlação significativa a $5 \%$.

Portanto, elege-se o modelo A, SARIMA $(2,1,0) \times(0,1,1) 12$, para a previsão do ICMS do Paraná por este apresentar resíduo do tipo ruído branco e coeficientes significativos.

\section{PREVISÃO DO ICMS A PARTIR DO MODELO SARIMA $(2,1,0) X(0,1,1) 12$}

A partir da identificação do modelo SARIMA $(2,1,0) \times(0,1,1) 12$ para a variável D1D12_ ICMS, foram estimados os parâmetros do modelo utilizando os softwares Eviews 5.0 (2004) e Statgraphics (2007). Os resultados são apresentados na Tabela 4.

TABELA 4 - ESTIMAÇÃO DO MODELO SARIMA $(2,1,0) \mathrm{X}(0,1,1) 12$

\begin{tabular}{l|rrrrr}
\hline \multicolumn{7}{c}{ Saída do software Eviews* } \\
\hline \multicolumn{1}{c}{ Parâmetro } & Coeficiente & Erro-padrão & t-Stat & Probabilidade \\
\hline C & 9765966 & 6383551 & 1529864 & 0.1284 \\
AR(1) & -0.5373 & 0.0827 & -6493108 & 0.0000 \\
AR(2) & -0.2695 & 0.0827 & -3258919 & 0.0014 \\
MA(12) & -0.850437 & 0.032219 & -2639582 & 0.0000 \\
\hline \multicolumn{5}{r}{ Saída do software Statgraphics** } \\
\hline \multicolumn{7}{c}{ Parâmetro } & Coeficiente & Erro-padrão & t-Stat & Probabilidade \\
\hline Mean & 987,792 & 613,970 & 1,609 & 0,110 \\
AR(1) & $-0,533$ & 0,083 & $-6,438$ & 0,000 \\
AR(2) & $-0,275$ & 0,083 & $-3,327$ & 0,001 \\
SMA(1) & 0,8323 & 0,0358 & 23,189 & 0,000 \\
\hline
\end{tabular}

FONTE: Saída do software Statgraphics (2007) e Eviews 5.0 (2004).

NOTA: $(*)$ estimação com 8 iterações; (**) estimação com 5 iterações.

As diferenças entre os resultados dos softwares decorrem dos algoritmos utilizados na maximização da função de verossimilhança e do número de iterações. Observa-se, porém, que as estimativas dos coeficientes são muito próximas e que as diferenças não afetaram os testes de significância dos parâmetros a 5\%.

Em modelos da classe ARIMA, a projeção que minimiza o erro ao quadrado é a esperança condicional da variável aleatória para o período $t+h$, dadas as observações passadas da série (MORETTIN; TOLOI, 2006, p. 223). Assim, para estimar a esperança condicional da variável aleatória para o período $t+h$, deve-se proceder de forma recursiva, estimando as esperanças para os períodos $t+1, t+2$, e assim por diante até $t+h$. Foram, então, estimadas as esperanças condicionais para a variável D1D12_ICMS. Estas previsões apresentam variância crescente entre agosto de 2011 e julho de 2012. Após o décimo segundo mês, a projeção con- 
verge para a média porque os termos AR têm função de autocorrelação com decaimento exponencial, enquanto o termo MA contribui para a previsão somente até o décimo segundo mês (MORETTIN; TOLOI, 2006, p. 223).

Para realizar a previsão da série de ICMS, as diferenças aplicadas na série ICMS, que geraram a série D1D12_ICMS, devem ser desfeitas. Deve-se realizar a seguinte operação para obter a série não diferenciada:

$$
Y_{t}=Y_{t-12}-Y_{t-13}+Y_{t-1}+Z_{t}
$$

Procedendo desta forma sobre os valores previstos para a série D1D12_ICMS, as arrecadações mensais previstas para o ICMS do Paraná, no período de agosto de 2011 a julho de 2012, são mostradas na Tabela 5.

TABELA 5 - PROJEÇÃO DO ICMS PARA O HORIZONTE DE 12 MESES

\begin{tabular}{lr|r|r}
\hline \multicolumn{1}{c|}{ Ano e mês } & Previsão & Limite inferior 95\% & Limite superior 95\% \\
\hline 2011.08 & 1316668,2 & 1231654,0 & 1401682,5 \\
2011.09 & 1354211 & 1172867 & 1535554,5 \\
2011.10 & 1356441 & 1078764 & 1634116,5 \\
2011.11 & 1387412 & 1012649 & 1762173,4 \\
2011.12 & 1412067 & 939991,3 & 1884140,5 \\
2012.01 & 1435468 & 866080,3 & 2004854,5 \\
2012.02 & 1362980 & 696264,6 & 2029694,5 \\
2012.03 & 1400103 & 636054,6 & 2164149,3 \\
2012.04 & 1447590 & 586209,6 & 2308969 \\
2012.05 & 1448145 & 489431,6 & 2406857 \\
2012.06 & 1452815 & 396769,3 & 2508860,7 \\
2012.07 & 1455009 & 301630,3 & 2608387,7 \\
\hline
\end{tabular}

FONTE: Saída do software Statgraphics (2007).

Com base no modelo SARIMA $(2,1,0) \times(0,1,1) 12$, espera-se que a arrecadação de ICMS que, em julho de 2011 foi de 1.267.566.000 de reais ${ }^{3}$, aumente 13,2\% até janeiro de 2012, quando atingirá 1.435.468.000 de reais. Em fevereiro, a arrecadação de ICMS será 5\% menor que no mês anterior, mas apresentará aumento acelerado entre fevereiro e abril, mês em que a arrecadação esperada atinge 1.447.590.000 de reais. Para os meses de maio a julho, espera-se que a arrecadação tenha pouca variação, aumentando 0,4\% e atingindo o nível de 1.455.009.000 de reais.

\section{CONCLUSÕES}

Neste artigo, buscou-se realizar previsão para a série de ICMS do estado do Paraná com base na metodologia Box-Jenkins. Para deflacionar a série, utilizou-se o IGP-DI referido a

3 Os valores em reais referidos neste artigo estão em moeda constante pelo IGP-DI (julho de $2011=100 \%$ ). 
julho de 2011. Por meio de testes de estacionariedade do tipo Dickey-Fuller, verificou-se que a série se torna estacionária tomando-se a primeira diferença juntamente com a diferença sazonal de ordem 12. Desta forma, criou-se a série D1D12_ICMS, a qual foi utilizada para a estimação de modelos da classe SARIMA (p,d,q)x(P,D,Q)12. Analisando os resíduos dos modelos que apresentaram coeficientes significativos a 5\%, concluiu-se que o melhor modelo para previsão é o SARIMA $(2,1,0) \times(0,1,1) 12$.

A identificação de sazonalidade na série de ICMS do estado do Paraná corrobora o trabalho de Liebel (2004). Porém, o mencionado autor utilizou a série de ICMS do período de janeiro de 1997 a dezembro de 2002, e não empregou teste para raiz unitária sazonal. Desta forma, a divergência entre o modelo identificado por Liebel (2004) e o identificado neste artigo decorre de serem distintos os períodos de análise e do fato de não ter sido aplicada diferenciação sazonal no primeiro.

Neste artigo, a partir do modelo SARIMA $(2,1,0) \times(0,1,1) 12$, realizaram-se previsões para o horizonte de 12 meses para a série diferenciada D1D12_ICMS. Para obter a previsão para a série de arrecadação do ICMS foi necessário desfazer as diferenças da série regredida. Procedendo desta forma, obteve-se a previsão para o ICMS, segundo a qual a arrecadação crescerá 13,2\% entre julho de 2011 e janeiro de 2012, diminuirá 5\% entre janeiro e fevereiro de 2012, e aumentará 6,7\% entre fevereiro e julho de 2012.

Sabe-se que o horizonte de previsão relevante dos modelos ARIMA é o curto prazo. A introdução de termos sazonais apenas aumenta este horizonte de previsão de acordo com a ordem dos termos AR e MA sazonais. Desta forma, dada a importância da arrecadação do ICMS, sugere-se que estas previsões de ICMS sejam atualizadas periodicamente.

\section{REFERÊNCIAS}

BOX, G.; JENKINS, G. Time series analysis: forecasting and control. San Francisco: Holden-Day, 1970.

CAMARgOS, A. A. B. Modelos de previsão de arrecadação tributária do estado de São Paulo: ICMS, IPVA, ICMD e Taxas. 170 f. Dissertação (Mestrado Profissional em Economia) - IBMEC, São Paulo, 2008.

CASTANHO, B. J. S. Modelos para previsão de receitas tributárias: o ICMS do estado do Espírito Santo. Dissertação (Mestrado em Economia) - Centro de Ciências Jurídicas e Econômicas, Universidade Federal do Espírito Santo, Vitória, 2011. Disponível em: <http://ppgeconufes.weebly.com/uploads/5/7/8/8/5788000/diss_01_2011.pdf >. Acesso em: 8/9/2011.

CORVALÃO, E. D. Previsão da arrecadação do imposto sobre circulação de mercadorias e serviços em Santa Catarina: aplicação da abordagem geral para específico em modelos dinâmicos. Dissertação (Mestrado em Engenharia da Produção) - Centro Tecnológico, Universidade Federal de Santa Catarina, Florianópolis, 2002. 
CRUZ, C. C. S. Análise de séries temporais para previsão mensal do ICMS: o caso do Piauí. Dissertação (Mestrado Profissional em Economia) - Centro de Aperfeiçoamento de Economistas do Nordeste, Universidade Federal do Ceará, Fortaleza, 2007. Disponível em: $<$ http://www.caucaianet.com/corecon-ce/imagens/cristovamcolombodossantoscruz.pdf $>$. Acesso em: 8/9/2011.

DICKEY, D. A.; HASZA D. P.; FULLER W. A. Testing for unit roots in seasonal time series. Journal of the American Statistical Association, v. 79, n. 386, jun. 1984. Disponível em: <http://www.jstor.org/stable/2288276>. Acesso em: 8/9/2011.

GUARAGNA, P. R. S.; MELLO, M. R. Um modelo de previsão para a arrecadação do ICMS. 46 f. Monografia (VII Prêmio Tesouro Nacional) - Secretaria do Tesouro Nacional, 2002.

IPARDES. Crescimento, reestruturação e competitividade industrial no Paraná: 1985-2000. Disponível em: < http://www.ipardes.gov.br/webisis.docs/crescimento_industrial.pdf>. Acesso em: 8/9/2011.

IPEADATA. IGP-DI Mensal. Disponível em: <http://www.ipeadata.gov.br/>. Acesso em: 4/9/2011(a).

IPEADATA. Imposto sobre a circulação de mercadorias (ICMS) - Paraná (PR). Disponível em: <http://www.ipeadata.gov.br/>. Acesso em: 4/9/2011(b).

LIEBEL, M. J. Previsão de receitas tributárias - o caso do ICMS no estado do Paraná. Dissertação (Mestrado Profissionalizante em Engenharia) - Escola de Engenharia, Universidade Federal do Rio Grande do Sul, Porto Alegre, 2004.

LIEBEL, M. J.; FOGLIATTO F. S. Método para previsão de receita tributária. In: ENCONTRO NACIONAL DE ENGENHARIA DE PRODUÇÃO, 25., 2005. Porto Alegre. Anais... Porto Alegre: ENEGEP, 2005.

MARQUES, C. A. G.; UCHÔA, C. F. A. Estimação e previsão do ICMS na Bahia. Revista Desenbahia, Salvador, v. 3, n. 5, p. 195-211, 2006.

MONTAÑÉS, A.; SANSÓ, A. The Dickey-Fuller test family and changes in the seasonal pattern. Annales D'Économie et de Statistique, n. 61, 2001. Disponível em: <http://annales. ensae.fr/anciens/n61/vol61-06.pdf>. Acesso em: 8/9/2011.

MORETTIN, P. A.; TOLOI, C. M. C. Análise de séries temporais. 2. ed. São Paulo: Edgard Blucher, 2006.

PASSOS, J. J.; RAMOS, E. M. L. S.; ALMEIDA, S. S. Utilização de modelos ARIMA para previsão da arrecadação de ICMS do estado do Pará. Disponível em: < http://www.ufpa.br/abe/ programacao/resumos/com-jairopasso2.pdf>. Acesso em: 4/9/2011.

PIERSE, R. G. Economic forecasting. Lecture 6: seasonality. Disponível em: <http://www.shs. surrey.ac.uk/economics/rpierse/ec451/bf6.pdf>. Acesso em: 8/9/2011.

REZENDE, F. ICMS: como era, o que mudou ao longo do tempo, perspectivas e novas mudanças. Cadernos Fórum Fiscal, Brasília, n. 10, p. 1-50, 2009.

SANTOS, A. V.; COSTA, J. H. F. Análise de modelos de séries temporais para a previsão mensal do ICMS do estado do Maranhão para o ano de 2008. Cadernos IMESC 6, São Luís, 2008.

STN. Secretaria do Tesouro Nacional. Execução orçamentária dos estados: 1995-2008. Disponível em: <http://www.tesouro.fazenda.gov.br/estados_municipios/download/exec_orc_estados.xls>. Acesso em: 8/9/2011. 
